\title{
Contribution of a Smart Transformer in the Local Primary Control of a Microgrid
}

\author{
T. L. Vandoorn, W. Willems, J. D. M. De Kooning, J. Van de Vyver and L. Vandevelde \\ Department of Electrical Energy, Systems \& Automation, \\ Ghent University, Ghent, Belgium, Tine.Vandoorn@UGent.be.
}

\begin{abstract}
In order to enable an easy participation of microgrids in the electricity markets, the smart transformer (ST) concept has been developed. The ST controls the power exchange between a microgrid and the utility network by only controlling its microgrid side voltage, instead of the conventional arrangement where new set points are communicated to all microgrid elements. When the voltage-based droop (VBD) control is implemented in the DG units, loads and storage elements, all microgrid units automatically respond to this change of microgrid voltage by altering their power output or consumption. However, this reference value of power exchange is dependent on (day-ahead) predictions of both consumption and (renewable) power generation. Hence, when these predictions prove to be inaccurate, the ST will still control the power exchange, but with consequently large variations of the microgrid voltage from its nominal value. It is suggested to take the real-time microgrid voltage into account when determining the reference power of the ST. This is presented in this paper by extending the ST's control strategy with a VBD control, such that the ST can contribute in the primary control. Simulations are included to analyze this primary control of the ST combined with VBD control of the other microgrid elements.
\end{abstract}

Index Terms-Microgrid, droop control, primary control, onload tap changing transformer, distributed generation units

\section{INTRODUCTION}

I Recently, there has been a considerable increase in the amount of distributed generation (DG) units in the electric power system, a large part of which use renewable energy sources. The main reasons for this change are the uncertain prices of fossil fuels, concerns about climate change and about the dependency of energy supply to other countries and the liberalization of the energy markets. Despite the numerous advantages these small-scale units offer, the current fit-andforget approach of connecting them to the electrical network is not a sustainable option. The distribution system is increasingly being confronted with congestion and voltage problems, which limits the further penetration of DG. Hence, a more coordinated approach for integrating DG in the distribution networks is required. Microgrids are designed to provide this coordination by aggregating generators, loads and storage elements [1]. They are likely to play a key role in the evolution of the smart grid [2]. In this sense, the smart grid can emerge as a system of integrated smart microgrids [3].

Although microgrids can operate both in grid-connected and islanded mode, the normal operating mode is the grid-

The work of T. Vandoorn is financially supported by a Fellowship of the FWO-Vlaanderen (Research Foundation - Flanders, Belgium). This research has been carried out in the frame of the Interuniversity Attraction Poles Programme initiated by the Belgian Science Policy Office (IAP-VII-02). The research of J. D. M. De Kooning is funded by the Special Research Fund (BOF) of Ghent University (Belgium) connected mode. In this mode, microgrids present themselves to the utility network as controllable entities. It enables the grid elements to achieve scaling benefits. For example, firstly, units that are otherwise too small can participate in the electricity markets through aggregation in microgrids. Secondly, by aggregating different kinds of units in a microgrid, the flexibility increases. This enables to deal with uncertainties of the predictions. Hence, microgrids can provide a significant value by operating in the electricity markets.

The power exchange between a grid-connected microgrid and the overlaying utility network is conventionally controlled in a secondary (or tertiary) control strategy that communicates new set points to the microgrid elements, i.e., loads, DG units and storage elements [4]. Opposed to a primary controller, which operates fast and automatically, a secondary controller operates slower and can take advantage of (smart grid) communication. The voltage-based droop (VBD) control of [5] is a primary controller developed for islanded microgrids. In [6], it is shown that, firstly, by combining this VBD control strategy with a smart transformer (ST), the microgrid operates in a virtually islanded mode. This means that the microgrid elements can use the same control strategy both in gridconnected and islanded mode. Secondly, the control strategy of the ST is compatible with the control of the loads and generators, because they are all voltage-based. Thirdly, for controlling the power exchange, only communication between the utility grid and the ST is required. The microgrid adapts automatically to changes in the power transfer. Therefore, the usage of an ST enables one of the main advantages of microgrids, namely that the microgrid can be seen as a controllable entity from the utility grid's point of view [7].

The smart transformer controls the power exchange to the value agreed upon in the electricity markets by controlling its microgrid-side voltage. However, this reference value is dependent on (day-ahead) predictions of both consumption and (renewable) power generation. Hence, when these predictions prove to be inaccurate, the ST will still control the power exchange to the pre-agreed reference value, possibly leading to a large deviation of the microgrid voltage from its nominal value. Therefore, in this paper the real-time microgrid voltage is taken into account when determining the reference power of the ST, such that the ST can contribute in the primary control. Hence, a voltage-based droop control, similar to the VBD control of the other microgrid elements, is implemented in the ST.

In $\S$ II, the conventional approach for microgrid market participation, with communication of new set points to all grid elements, is summarized. To avoid this communication burden, the ST concept has been introduced. A review of the 


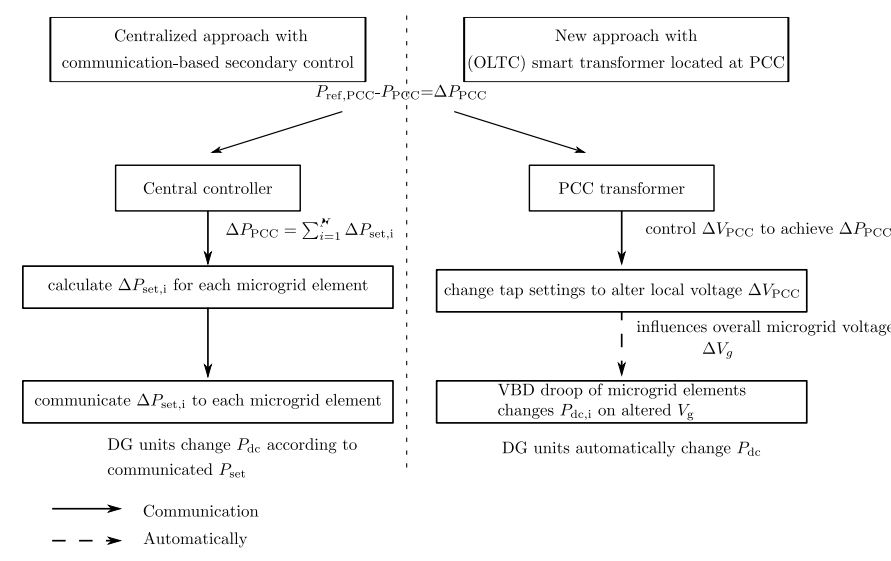

Fig. 1. Smart transformer versus central control to change power export from microgrid to utility network

ST operation is given in $\S$ III. Next, a primary VBD control is included in the ST in $\S$ IV. Some simulations are provided in $\S \mathrm{V}$ in order to clarify the operation of the ST with primary VBD control in a grid-connected microgrid.

\section{OVERVIEW OF SMART TRANSFormer CONCEPT}

\section{A. Microgrid market participation}

A requirement for the market participation of a microgrid is that the power exchange between the microgrid and the utility network can be controlled to the reference value which is negotiated in the markets, e.g., on a day-ahead basis (market arrangements inside the microgrid are not handled in this paper). For example, when the market price is high, the microgrid will consume as little power as possible from the utility network, or even export when possible. The microgrid will try to shift its consumption to times of a low market price. It will also shift its consumption to mitigate load peaks.

Conventionally, the power exchange is controlled by a microgrid central controller that communicates new set points to all grid elements, in such a way that the total consumption/production complies with the reference power exchange, as depicted in Fig. 1 [6].

\section{B. Smart transformer concept}

In order to reduce the required communication data for controlling the power exchange, the smart transformer concept has been presented in [6]. Instead of exchanging new set points with all microgrid elements, only the ST needs to acquire the set point. As depicted in Fig. 2, the ST is a controlled tap changing transformer that is connected at the point of common coupling (PCC) of the microgrid. The transformer is smart in the sense that its control strategy is able to control the power exchange to a set value by controlling its microgridside voltage.

In medium-voltage networks, on-load tap changing transformers (OLTCs) are sometimes already in place. Hence, controlling these as smart transformers requires only little modifications. In the lower voltage networks, most PCC transformers are manual tap changing transformers, from which the voltage can only be controlled offline and not automatically. This puts a significant stress on the electrical grids which

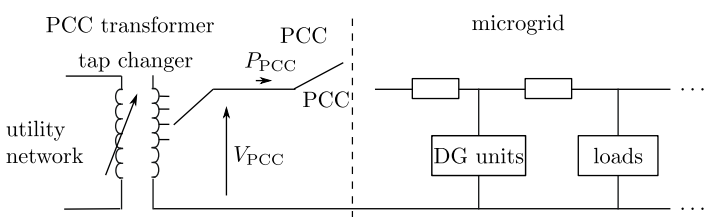

Fig. 2. Smart transformer located at the PCC of a microgrid

face an increased penetration of DG units. Historically, the planning of the low-voltage grids is based on a worst-case scenario ensuring that in case of maximum consumption, the voltage does not drop below the lower voltage limit. Therefore, many tap changing transformers at the beginning of the low-voltage lines are set somewhat above the nominal voltage. However, with the increasing degree of DG units, the risk of overvoltage occurrence becomes higher. Also, the planning becomes more difficult because of the larger voltage variations (e.g., sunny day versus night times). Hence, the ability of automatically changing the tap settings becomes more interesting as it is more effective, faster and cheaper to implement than the conventional approach of investing in the grid assets, such as installing more grid lines. Therefore, in the future, it is expected to become more beneficial to install an OLTC. Moreover, it is well-known that a lot of the assets in distribution networks are end-of-life and have to be replaced anyhow in the following years. Hence, the manual transformers can gradually be upgraded to OLTCs.

The OLTC transformer, with smart control strategy, i.e., the smart transformer, controls the PCC power $\left(P_{\mathrm{PCC}}\right)$ by controlling the microgrid side voltage $\left(V_{\mathrm{PCC}}\right)$. The control algorithm of the ST is based on the following discrete PI controller to force $P_{\mathrm{PCC}}$ to $P_{\mathrm{PCC}, \text { ref }}$ [6]:

$$
V_{\mathrm{PCC}, \mathrm{k}}=V_{\mathrm{PCC}, \mathrm{k}-1}+\left(e_{k}-e_{\mathrm{k}-1}\right) K_{1}+e_{k} K_{2}
$$

with $e_{k}=P_{\mathrm{PCC}, \mathrm{ref}, \mathrm{k}}-P_{\mathrm{PCC}, \mathrm{k}} ; V_{\mathrm{PCC}, \mathrm{k}}$ the STs secondary voltage; and $k$ the discrete time step. Power transfer from utility grid to microgrid has a positive sign $\left(P_{\mathrm{PCC}}>0\right)$, while export from the microgrid into the utility has a negative sign of $P_{\mathrm{PCC}}$. A higher power transfer $P_{\mathrm{PCC}}$ can be achieved by increasing $V_{\mathrm{PCC}}$, while of course, a lower $V_{\mathrm{PCC}}$ has the opposite effect. A consequence of a higher $V_{\mathrm{PCC}}$ is that the overall voltage in the microgrid increases. As discussed below, the VBD controllers of the DG units for example will decrease the dc-power in response to the increased terminal voltage. In this way, the DG units automatically deliver less power when the power import into the microgrid is increased by setting a higher $V_{\mathrm{PCC}}$ with the $\mathrm{ST}$.

The VBD control strategy, illustrated in Fig. 3, has originally been presented for ensuring a stable operation of lowvoltage islanded microgrids [5]. For the active power control of the DG units, this VBD controller consists of a combination of a $V_{\mathrm{g}} / V_{\mathrm{dc}}$ droop controller and a $P / V_{\mathrm{g}}$ droop controller, with $V_{\mathrm{dc}}$ the dc-link voltage and $V_{\mathrm{g}}$ the terminal voltage of the DG unit. The former enables power balancing of the DG unit's ac and dc side and an effective usage of the allowed tolerance on the variations of terminal voltage from its nominal value for grid control. It is based on the dc-link capacitor of the converter taking the role of the rotating inertia in conventional grid control [9]. In this way, changes in the dc-link 
voltages indicate a difference between the ac-side (electrical) and dc-side (mechanical) power, which is analogous as the frequency changes in the conventional power systems. The $P / V_{\mathrm{g}}$ droop controller enables to avoid voltage limit violation and is combined with constant-power bands that delay the active power changes of the renewables (wide constant-power band) compared to those of the dispatchable DG units (small constant-power band) to more extreme voltages (Fig. (4). For the loads and storage elements, a similar voltage-based control strategy is presented in [8].

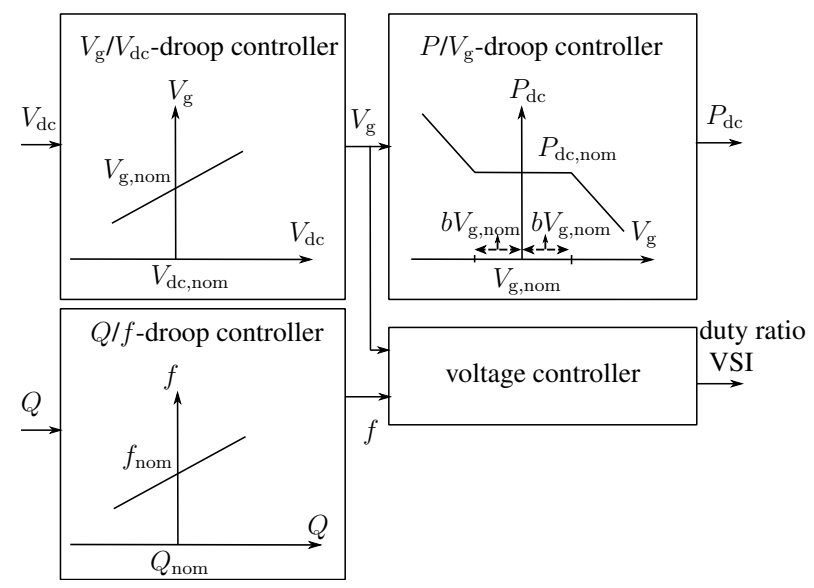

Fig. 3. Voltage-based droop control strategy
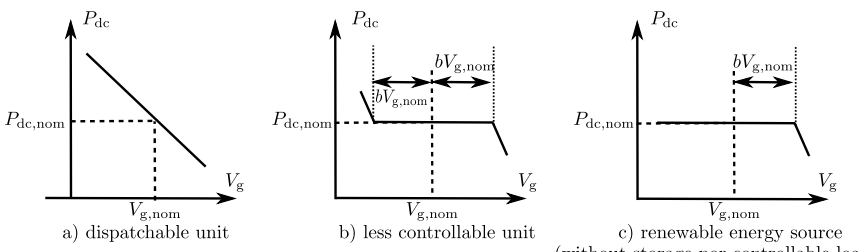

Fig. 4. Constant power bands of dispatchable versus less-dispatchable DG units

As all microgrid elements, the loads, storage elements and DG units, react on a changing terminal voltage, the VBD control can be used in a grid-connected microgrid with ST, which is also voltage-controlled to alter the PCC power. For example, when the PCC power drawn from the utility network is higher than its pre-agreed value $\left(P_{\mathrm{PCC}, \mathrm{ref}}\right)$, the smart transformer will lower $V_{\mathrm{PCC}}$. The microgrid DG units react on this voltage drop by increasing their output power, hence, increasing $\left|P_{\mathrm{PCC}}\right|$. Similarly, the active loads can change their consumption based on their terminal voltage [8]. In this way, the PCC power can be controlled by the smart transformer, without the need to communicate new set points to all grid elements as they automatically react. A second advantage is that in this way, a virtual islanded mode is achieved. The utility grid is not seen as a slack bus, but is conceived as a constant-power load/generator, i.e., the power agreed upon in the markets.

Summarized, the power transfer $P_{\mathrm{PCC}}$ is altered by communicating $P_{\mathrm{PCC} \text {,ref }}$ to the $\mathrm{ST}$. This ST uses a tap changer to influence its microgrid-side voltage $V_{\mathrm{PCC}}$. This directly influences the active power in the microgrid because the active power control of both the generators and the active loads is voltage-triggered [5], [8]. Hence, these units automatically change their input/output power dependent on the grid voltage and thus, dependent on $V_{\mathrm{PCC}}$. Therefore, $P_{\mathrm{PCC} \text {,ref }}$ only needs to be communicated to a single unit and the rest of the microgrid elements adapt without communication. In this way, the microgrid can be exploited as a controllable entity within the electrical network for the primary control.

\section{SMART TRANSFORMER CONTRIBUTION IN THE PRIMARY CONTROL}

As illustrated in [6], the ST has a significantly added value in controlling the PCC power to the reference value agreed upon in the electricity markets. However, the paper also suggests that the reference power exchange can be altered depending on the instantaneous status of the microgrid as well. The reason is that the reference PCC power in the markets is agreed dependent on (day-ahead) predicted values of load and generation, which may be inaccurate in some time periods. If the prediction significantly deviates from the real-time value, controlling the power exchange to the reference value may become hard. For example, if the generation (photovoltaic, wind) is significantly higher than the predicted value and simultaneously, in the markets, a large injection from power into the microgrid $\left(P_{\mathrm{PCC}, \mathrm{ref}}\right)$ is agreed, this will lead to high microgrid voltages. The primary VBD controller in the microgrid, which is a fast automatic control strategy taking care of the stability of the network, will react. In an automatic priority, the dispatchable DG units will lower their generated power, next, the storage elements and some loads and later the renewables will take appropriate actions to ensure a stable microgrid operation and a proper voltage quality in the microgrid.

When the power exchange is dependent on both the agreed value in the markets and the microgrid state, the ST can also contribute in this primary control and take a place in the priority list for power changes described above. In this way, power curtailment of the renewable energy sources or load control can be avoided if the ST reacts first on voltage changes (i.e., lower constant-power band $b$ ). Fig. 5 shows the VBD control strategy of the ST. This VBD controller changes the reference power exchange $P_{\mathrm{PCC}, \text { ref }}$ dependent on the terminal voltage $V_{\mathrm{g}}$ of the ST (i.e., the measured $V_{\text {PCC }}$ ) by means of a droop controller. For terminal voltages inside the constant-power band (with width $2 b \cdot V_{\mathrm{g}, \text { nom }}$ ), the reference power exchange is determined by the markets, i.e., through the secondary controller $P_{\mathrm{PCC}, \mathrm{ref}, \mathrm{market}}$. Only for higher deviations of the voltage compared to its nominal value, the VBD controller intervenes. The ST should take action before the renewables need to. Therefore, the ST is equipped with a constant power band that is smaller than that of the renewables.

\section{PROOF OF CONCEPT}

The simulation case depicted in Fig. 6 is considered. This case has the same configuration as that in [6] but, here, the first DG unit, G1, generates three times more power than its predicted value $\left(I_{\mathrm{dc}, 1}=4.5 \mathrm{~A}\right.$ instead of $1.5 \mathrm{~A}$ in the former paper). This large output power combined with an import into 


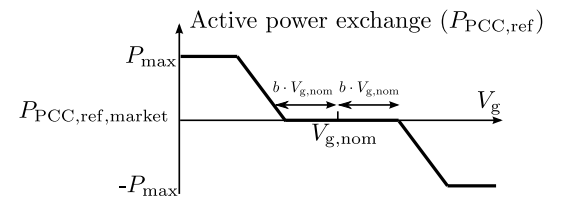

Fig. 5. Smart transformer with voltage-based droop control to alter $P_{\mathrm{PCC}, \text { ref }}$

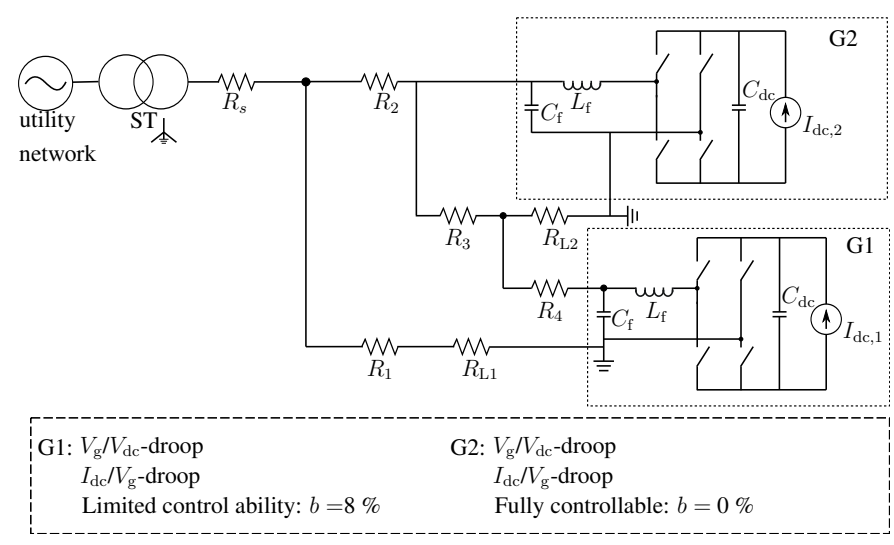

Fig. 6. Microgrid configuration for proof of concept using smart transformer: single-line diagram

the microgrid and a relatively low load lead to high microgrid voltages. These values are chosen in order to clarify the effect of the primary VBD control of the ST in avoiding these high voltages and the power curtailment of the renewable energy source G1. The simulations are done in Matlab/Simulink with the PLECS library for simulations up to the level of the converter switches. The simulation parameters are summarized in Table 1 Virtual output impedance behavior $r_{\mathrm{v}}$ according to [10] is included in the DG units and the line impedances are purely resistive. The latter is a valid assumption as generally, the low-voltage microgrid lines are mainly resistive, and this effect is increased by the resistive virtual output impedance.

The microgrid consists of one ST located at the PCC, two DG units and two loads $R_{\mathrm{L}, 1}$ and $R_{\mathrm{L}, 2}$, as depicted in Fig. 6 . Both DG units apply the $V_{\mathrm{g}} / V_{\mathrm{dc}}$ droop and $I_{\mathrm{dc}} / V_{\mathrm{g}}$ droop control strategies, the latter being similar tot he $P / V_{\mathrm{g}}$ droop controller. The first DG unit, G1, has a wide constant-power band $2 b$ of $16 \%$, representing a slightly controllable DG unit, e.g., a renewable energy source with small storage capabilities. This paper illustrates the transient response, hence, e.g., PV panels and wind turbines are modeled as dc-current sources. For longer time frames, modeling them as power sources proves to be more accurate. In this paper, the changes of, e.g., solar irradiation, changing $I_{\mathrm{dc}}$ are not taken into account. The second DG unit, G2, has a constant-power band $2 b$ of $0 \%$, thus, represents a dispatchable unit. The nominal input dc-currents are 4.5 A and $3 \mathrm{~A}$ for G1 and G2 respectively.

Three cases will be analyzed. First, the ST controls the power exchange $P_{\mathrm{PCC}}$ to a pre-determined fixed value. Next, VBD control is included in the ST, so that the power exchange can be altered by this primary controller. Third, the VBD controller of the ST is more stringent.

- No VBD control in smart transformer. $P_{\mathrm{PCC}, \text { ref }}=$
TABLE I

SYSTEM PARAMETERS. THE LINE PARAMETERS ARE BASED ON BAXB CABLES WITH PHASE RESISTANCE $0.41 \Omega / \mathrm{km}$ AND NEUTRAL CONDUCTOR WITH $0.71 \Omega / \mathrm{km}$ AND $R_{\text {phase }} / X \approx 5$.

\begin{tabular}{|l|l||l|l|}
\hline parameter & value & parameter & value \\
\hline$L_{\mathrm{f}}$ & $2 \mathrm{mH}$ & $C_{\mathrm{f}}$ & $3 \mu \mathrm{F}$ \\
$R_{1}$ & $0.05 \Omega$ & $C_{\mathrm{dc}}$ & $1.5 \mathrm{mF}$ \\
$R_{2}$ & $0.3 \Omega$ & Droop of $V_{\mathrm{g}} / V_{\mathrm{dc}}$ & $0.5 \mathrm{~V} / \mathrm{V}$ \\
$R_{3}$ & $0.3 \Omega$ & Droop of $Q / f$ & $5 \mathrm{e}^{-5} \mathrm{~Hz} / \mathrm{VAr}$ \\
$R_{4}$ & $0.3 \Omega$ & Droop of $I_{\mathrm{dc}} / V_{\mathrm{g}}$ & $I_{\mathrm{dc}, \text { nom }} / 50$ \\
$R_{s}$ & $0.5 \Omega$ & $f_{\text {nom }}$ & $50 \mathrm{~Hz}$ \\
$R_{\mathrm{L} 1}$ & $33 \Omega$ & $Q_{\mathrm{nom}}$ & $0 \mathrm{VAr}$ \\
$R_{\mathrm{L} 2}$ & $33 \Omega$ & $I_{\mathrm{dc}, \text { nom }, 1}$ & $4.5 \mathrm{~A}$ \\
$r_{\mathrm{v}}$ & $3 \Omega$ & $I_{\mathrm{dc}, \text { nom }, 2}$ & $3 \mathrm{~A}$ \\
$V_{\mathrm{dc}, \text { nom }}$ & $450 \mathrm{~V}$ & $V_{\mathrm{g}, \text { nom }}$ & $230 \mathrm{~V} \mathrm{rms}$ \\
\hline
\end{tabular}

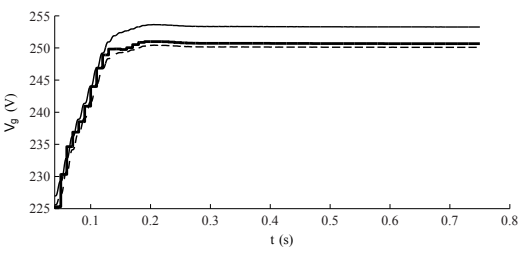

(a) Microgrid rms voltage

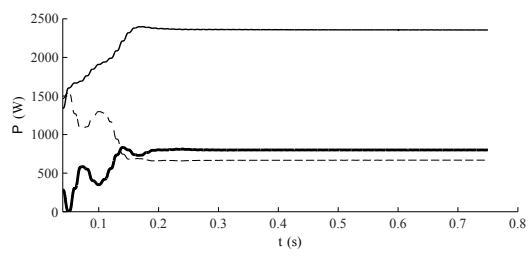

(b) Active power to the electrical network

Fig. 7. Influence of smart transformer without VBD control $(-=\mathrm{G} 1$; --$=\mathrm{G} 2 ;-=$ smart transformer)

$P_{\mathrm{PCC}, \text { ref,market }}=800 \mathrm{~W}$ in Fig. 7) This simulation shows that the ST can track a reference active power by changing its microgrid-side voltage. Also, because of the voltage-based control of the DG units and the ST, the microgrid automatically responds to changes of the ST. The microgrid imports relatively much power from the main grid, for example, because of low energy prices in the main grid and a (wrong) prediction of a low generation of the renewable energy source. However, as the actually delivered renewable energy is high, this leads to high voltages, close to the $10 \%$ overvoltage limit. The obtained steady-state results are summarized in Table II

- In order to avoid the critical voltages illustrated in the previous example, in Fig. 8, the ST is equipped with VBD control with $b=2 \%$ and the droop of the $P_{\mathrm{PCC}} / V_{\mathrm{g}}$ controller $K_{\mathrm{P}}$ equals $80 \mathrm{~W} / \mathrm{V}$. Hence, the ST also contributes in the primary control by lowering the power import into the microgrid. The simulations show a voltage closer to its nominal value and the power of the renewable source, DG1, is less curtailed. Hence, including primary VBD control in the ST is advantageous for both the 
TABLE II

OVERVIEW: INFLUENCE OF INCLUDING VBD CONTROL IN ST

\begin{tabular}{|c|ccc|}
\hline & no VBD & $\begin{array}{c}\text { ST control } \\
\text { VBD with } \\
\text { small droop }\end{array}$ & $\begin{array}{c}\text { VBD with } \\
\text { large droop }\end{array}$ \\
\hline$P_{1}(\mathrm{~W})$ & 2357 & 2719 & 2660 \\
$P_{2}(\mathrm{~W})$ & 668 & 927 & 1103 \\
$P_{\mathrm{PCC}}(\mathrm{W})$ & 800 & -2 & -247 \\
\hline$V_{1}(\mathrm{~V})$ & 253 & 248 & 243 \\
$V_{2}(\mathrm{~V})$ & 250 & 244 & 239 \\
$V_{\mathrm{PCC}}(\mathrm{V})$ & 251 & 242 & 236 \\
\hline$I_{\mathrm{dc}, 1}(\mathrm{~A})$ & 3.9 & 4.5 & 4.5 \\
$I_{\mathrm{dc}, 2}(\mathrm{~A})$ & 1.3 & 1.8 & 2.2 \\
\hline \multicolumn{4}{|r}{}
\end{tabular}

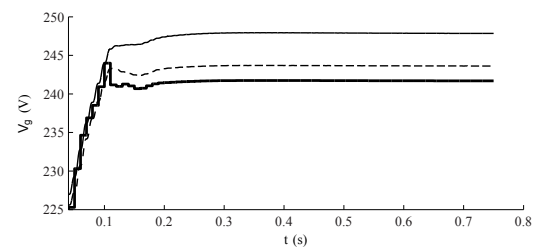

(a) Microgrid rms voltage

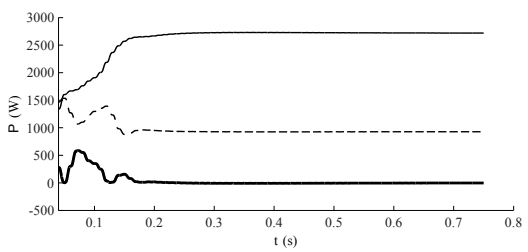

(b) Active power to the electrical network

Fig. 8. Influence of smart transformer with VBD control $(b=2 \%$ and $\left.K_{\mathrm{P}}=80 \mathrm{~W} / \mathrm{V}\right)(-=\mathrm{G} 1 ;----=\mathrm{G} 2 ;-=$ smart transformer $)$

voltage quality inside the microgrid as for the renewable energy capturing, while still, the ST can control the power exchange without inter-unit communication.

- In the third case in Table II the VBD controller in the ST has a larger droop, $b=2 \%$ and $K_{\mathrm{P}}=400 \mathrm{~W} / \mathrm{V}$. The microgrid even exports power to the utility network, the voltage is lower and DG1 can produce even more of the available renewable power as it is less curtailed. Hence, by setting the droop parameter $K_{\mathrm{P}}$, a trade-off is made between achieving a better voltage quality inside the microgrid (large $K_{\mathrm{P}}$ ) or better abiding by the predefined reference $P_{\mathrm{PCC}}\left(\right.$ lower $K_{\mathrm{P}}$ ).

In conclusion, by implementing a VBD controller in the ST, the PCC power can be altered dependent on the microgrid state as well as on the value agreed upon in the electricity markets. In this way, the contribution of the ST in the primary control can avoid curtailing renewable energy sources or addressing the load control. Dependent on the value of the droop, the amount of ST reaction is tuned. The width of the constantpower band determines which unit reacts first on a changing voltage. The following order, with increasing $b$, is suggested: (1) controllable DG units, (2) storage elements, (3) ST, (4) controllable loads, (5) less dispatchable DG units.

\section{CONCLUSiOns}

When voltage-based control is used inside the microgrid, a smart transformer (ST) can control the power exchange between the microgrid and the rest of the network by changing its microgrid side voltage. The microgrid elements automatically react. Hence, the ST does not require communication to the microgrid elements for controlling the power exchange to a reference value. It is suggested that the reference power exchange should be pre-determined in the electricity markets, as well as being dependent on the instantaneous local state of the microgrid. Only the former was already implemented in literature. Therefore, in this paper, the ST is extended with a control strategy enabling the local state of the microgrid to influence the reference power exchange, in an automatic manner. The ST is equipped with a control strategy similar to the voltage-based droop control of the DG units, loads and storage elements, that changes the ST's reference PCC power based on the microgrid voltage. Constant-power bands, similar to those of the other microgrid elements, are included such that the ST does not react on each voltage variation, but takes a place in the automatic priority list of the microgrid elements for their contribution in the primary control. In this way, the ST assists in the primary control of the microgrid and helps achieving a better voltage quality. The simulations verify the operation of this ST in a microgrid and show the advantages of including VBD control in the ST to alter the reference PCC power.

\section{REFERENCES}

[1] R. H. Lasseter, A. Akhil, C. Marnay, J. Stephens, J. Dagle, R. Guttromson, A. Meliopoulous, R. Yinger, and J. Eto, "The CERTS microgrid concept, white paper on integration of distributed energy resources," in California Energy Commission, Office of Power Technologies - U.S. Department of Energy, LBNL-50829, http://certs.lbl.gov, Apr. 2002.

[2] N. Lidula and A. D. Rajapakse, "Microgrids research: A review of experimental microgrids and test systems," Renewable and Sustainable Energy Reviews, vol. 15, no. 1, pp. 186-202, 2011.

[3] H. Farhangi, "The path of the smart grid," IEEE Power \& Energy Magazine, vol. 8, no. 1, pp. 18-28, Jan./Feb., 2010.

[4] J. Guerrero, P. Loh, M. Chandorkar, and T. Lee, "Advanced control architectures for intelligent microgrids - Part I: Decentralized and hierarchical control," IEEE Trans. Ind. Electron., vol. 60, no. 4, pp. 1254-1262, Apr. 2013.

[5] T. L. Vandoorn, B. Meersman, L. Degroote, B. Renders, and L. Vandevelde, "A control strategy for islanded microgrids with dc-link voltage control," IEEE Trans. Power Del., vol. 26, no. 2, pp. 703-713, Apr. 2011.

[6] T. L. Vandoorn, J. D. M. De Kooning, B. Meersman, J. Guerrero, and L. Vandevelde, "Voltage-based control of a smart transformer in a microgrid," IEEE Trans. Ind. Electron., vol. 60, no. 4, pp. 1291-1305, Apr. 2013.

[7] A. G. Tsikalakis and N. D. Hatziargyriou, "Centralized control for optimizing microgrids operation," IEEE Trans. Energy Convers., vol. 23, no. 1, pp. 241-248, Mar. 2008.

[8] T. L. Vandoorn, B. Renders, L. Degroote, B. Meersman, and L. Vandevelde, "Active load control in islanded microgrids based on the grid voltage," IEEE Trans. on Smart Grid, vol. 2, no. 1, pp. 139-151, Mar. 2011.

[9] T. L. Vandoorn, B. Meersman, J. D. M. De Kooning, and L. Vandevelde, "Analogy between conventional grid control and islanded microgrid control based on a global dc-link voltage droop," IEEE Trans. Power Del., vol. 27, no. 3, pp. 1405-1415, Jul. 2012.

[10] J. M. Guerrero, J. Matas, L. García de Vicuña, M. Castilla, and J. Miret "Decentralized control for parallel operation of distributed generation inverters using resistive output impedance," IEEE Trans. Ind. Electron. vol. 54, no. 2, pp. 994-1004, Apr. 2007. 